\title{
Case Report: Suspected Case of Stevens-Johnson Syndrome and Toxic Epidermal Necrolysis Overlap Due to Ursodeoxycholic Acid
}

\author{
Authors: \\ *Shatavisa Mukherjee, Debajyoti Saha, Shreyashi Dasgupta, Santanu \\ Kumar Tripathi \\ Department of Clinical and Experimental Pharmacology, School of Tropical Medicine, \\ Kolkata, India \\ *Correspondence to shatavisa100@gmail.com \\ Disclosure: $\quad$ The authors have declared no conflicts of interest. \\ Acknowledgements: The authors would like to acknowledge and support the untiring efforts and \\ contribution of the Pharmacovigilance Programme of India (PVPI) towards ensuring \\ better patient safety nationwide. Approval from the Institutional Ethics Committee \\ was obtained for publication of this case report. \\ Received: \\ 28.04.20 \\ Accepted: \\ 04.09 .20 \\ Keywords: \\ Case report, severe cutaneous adverse reactions (SCAR), Stevens-Johnson syndrome \\ (SJS), toxic epidermal necrolysis (TEN), ursodeoxycholic acid (UDCA). \\ Citation: \\ EMJ Dermatol. 2020;8[1]:96-99.
}

\section{Abstract}

Stevens-Johnson syndrome and toxic epidermal necrolysis are well-known severe cutaneous adverse reactions, with >100 medications previously implicated, most frequently sulfonamide antibiotics. Ursodeoxycholic acid (UDCA), normally present in human bile at a low concentration, is used for the treatment of various cholestatic disorders. Reports of UDCA causing cutaneous complications are, however, rare. The present report describes a suspected case of UDCA-induced Stevens-Johnson syndrome-toxic epidermal necrolysis overlap in a 24-year-old female, admitted with a whole-body maculopapular rash with oromucocutaneous ulceration and skin desquamation. The patient was managed with supportive care, including fluid and electrolyte replacement, corticosteroids, antibiotics, antihistamines, and intravenous Ig. Early identification, prompt intervention with effective care, and support are the key action points in these severe cutaneous adverse reactions.

\section{BACKGROUND}

Stevens-Johnson syndrome (SJS) and toxic epidermal necrolysis (TEN) are well-known severe cutaneous adverse reactions (SCAR). First reported in 1922, these reactions were initially thought to be infectious in nature, however, the concept has changed over the years. Although their aetiology is not fully understood, most

cases of SJS and TEN are now attributed to an immunologically mediated response to drug exposure, belonging to Type IV hypersensitivity. SJS-TEN refers to SCAR associated with widespread epidermal detachment and mucocutaneous involvement. Incidence of SJS and TEN is estimated to be 1.0-6.0 per million and 0.4-1.2 per million, respectively. ${ }^{2}$ Over 100 medications have been implicated in SJS and TEN, most frequently sulfonamide antibiotics, 
followed by nonsteroidal anti-inflammatory drugs and antigout drugs, particularly allopurinol.3,4 Risk of developing these SCAR after drug exposure appears to be greatest during the first few weeks of treatment initiation. These SCAR are characterised by fever, rash, and mucosal blisters. Diagnosis depends on the total body surface area involvement of detached/detachable skin lesions: $<10 \%, 10-30 \%$, and $>30 \%$ represent SJS, SJS-TEN overlap, and TEN, respectively. Both SJS and TEN can occur at any age, but appear to be more prevalent in adults, especially in older adults over 65 years. ${ }^{5}$ SJS-TEN overlap is slightly predominant in females compared with males $(3: 2){ }^{6}$

The dihydroxy bile acid ursodeoxycholic acid (UDCA) is used for the treatment of chronic cholestatic liver disorders. It is normally present in human bile at a low concentration of almost $3 \%$ of total bile acids. ${ }^{7}$ Regarded as a welltolerated drug, few safety concerns have been reported since its initial clinical use. Cutaneous complications are rare, though there have been reports of generalised rash, fixed drug eruptions, and lichen planus secondary to this drug. ${ }^{8,9}$ However, no prior reports of SCAR were available through an extensive literature search. The present report describes a suspected case of UDCA-induced SJS-TEN overlap.

\section{CASE REPORT}

A 24-year-old female, who was normotensive and euglycaemic, was admitted with whole-body maculopapular rash with oromucocutaneous erosions and skin desquamation. The patient had a history of viral hepatitis 1 year previous, and was symptom-free before the development of the rashes. No other viral infections were reported during this 1-year time frame. Ten days prior to the presenting features, she was commenced on UDCA $300 \mathrm{mg}$ twice daily (bid), along with a fixed-dose combination of omeprazole and domperidone once daily (ad), by a local physician owing to deranged liver function tests. On the seventh day of consumption of these medications, she presented with a rash, appearing first on the face and then slowly progressing all over the body. The rash was pruritic in nature and was followed by blister formation. The blisters were confined to the facial region, particularly involving the oromucocutaneous region. The blisters were followed by denudation of the skin (Figure 1). The patient was afebrile with no urinary abnormalities, and there were no genital lesions. She also described watery discharge from her eyes and had difficulty in opening her eyes and mouth.

The patient was admitted, with prompt cessation of all ongoing medications. She had a history of previous treatment with omeprazole and domperidone on multiple occasions, without any adverse event. However, skin biopsy and histopathology of the involved area was not performed, due to its unavailability in the rural setting of this case. Considering the clinical presentation and involved total body surface area, the patient was diagnosed with a suspected case of UDCA-induced SJS-TEN overlap. The temporality ruled out the probability of the reaction being related to any viral condition. Prognosis was assessed using SCORe of Toxic Epidermal Necrosis (SCORTEN) criteria, which conferred a score of 2 for the index case. The patient was managed with a short course of steroid therapy with qd dosing of dexamethasone for 3 days, intravenous fluid (normal saline) 8 hourly, cyclosporine $100 \mathrm{mg}$ bid, chlorhexidine mouthwash, calaminol lotion, hydroxyzine $25 \mathrm{mg}$ qd, moxifloxacin eye drops, and methylcellulose eye drops. The patient responded to this regimen and was discharged within 3 weeks. Her laboratory investigations were within normal limits.

Causality assessment of the reaction conferred it to be "probable", with a score of 5 using the Naranjo causality assessment algorithm, while the World Health Organization-Uppsala Monitoring Centre (WHO-UMC) causality assessment scale also graded it as "probable"; severity assessment using the Hartwig and Siegel Scale assessed it to be severe (Level 5). The event was reported under the Pharmacovigilance Programme of

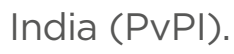

\section{DISCUSSION}

Approximately $45 \%$ of adverse drug reactions are manifested in the skin, with the majority being mild. However, drug-induced SCAR are not rare and are potentially life threatening. 


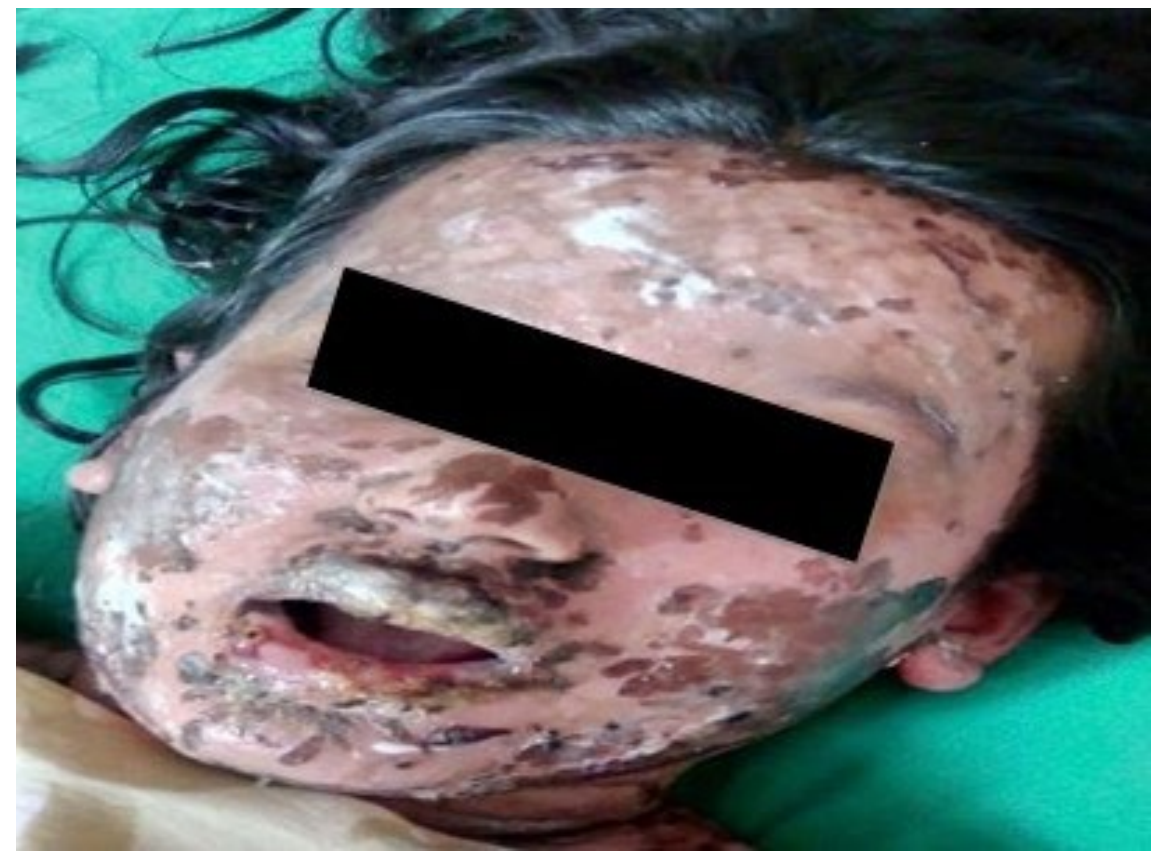

Figure 1: Oromucocutaneous ulceration and skin desquamation, consistent with Stevens-Johnson syndrome and toxic epidermal necrolysis overlap severe cutaneous adverse reaction.

These hypersensitivity reactions, including SJS and TEN, are primarily recognised as a dysregulation of cellular immunity caused by a release of various cytotoxic signals, including granulysin, perforin/granzyme B, and Fas/Fas ligands, which are activated by cytotoxic $T$ lymphocytes and natural killer cells. These SCAR differ from classical allergies as there is no classic sensitisation. As evidenced in the literature, mortality rates of SJS, SJS-TEN overlap, and TEN are $5-10 \%, 30 \%$, and $50 \%$, respectively. ${ }^{10}$ Patients usually give a history of constitutional symptoms, including fever, malaise, arthralgia, and sore throat. To start with, the lesions are erythematous to violaceous and purpuric macules, which coalesce to form patches. Targetoid lesions may be present. Mostly, the lesions initially involve the trunk and upper torso, which spread distally to involve the limbs, followed by skin exfoliation. Presentation of flaccid bullae is also common. SJS is characterised by involvement of $<10 \%$ body surface area, SJS-TEN overlap signifies 10\%$30 \%$ involvement and the most severe form of the spectrum, and TEN is characterised by involvement of $>30 \%$ body surface area. Mucosal inflammation (oral, ocular, and genitourinary) is nearly universal. Pseudo-Nikolsky and Asboe-
Hansen signs can be elicited in most cases. The hallmark findings include full-thickness epidermal necrosis, subepidermal bullae, and scanty inflammatory infiltrates in the papillary dermis." However, owing to logistic concerns, the present report could not describe the pathological findings of the affected area, which remains a limitation of this study. The clinical differentials of these SCAR include morbilliform drug rash, erythema multiforme, drug-induced linear IgA disease, acute generalised exanthematous pustulosis, acute graft-versus-host disease, drug reaction with eosinophilia and systemic symptoms syndrome, or staphylococcal scalded skin syndrome. UDCA, the suspect drug in this report, is virtually considered safe. However, rare reports of mild- to moderate-grade skin reactions have surfaced. Cutaneous manifestations such as lichenoid skin eruptions, itching, and prurigo

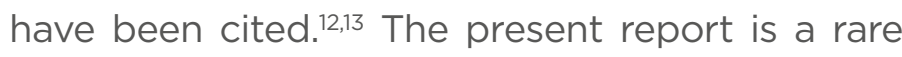
case of UDCA-induced SCAR. To the authors' knowledge, it is the first of its kind reported from this country. Though regarded to have negligible safety concerns, the responsible mechanism behind such a reaction may be due to the cytotoxic profile of this drug. ${ }^{8}$

An effective modality may be drug provocation testing by preparing a list of suspected drugs 
to which the patient has previously experienced drug reactions; however, such tests should be performed under strict medical supervision, preferably in a day care setting..$^{14}$ Several serum markers have also been explored, which can serve to detect an early TEN case and prognosticate its due progression. Of these many markers, few are soluble, including Fas ligand, granzyme B, soluble CD40 ligand, granulysin, serum high mobility group protein B1, serum lactate dehydrogenase, a-defensins $1-3$ in the blisters, $\mathrm{Bcl}-2$ expression in the dermal infiltrates, thymus and activation-regulated chemokine, and glutathione-S transferase-pi expression. IL-15 has been found to be useful in predicting severity and monitoring prognosis."

Patients with SJS or TEN are managed with supportive care, such as fluid and electrolyte replacement, corticosteroids, immunosuppressants, antibiotics, antihistamines, and intravenous Ig. Owing to multi-organ system involvement, complications can be varied depending on the reaction extent and point of therapeutic intervention, thus mandating early consultation with concerned specialties for ensuring safer patient outcomes.11,15 Early identification and prompt intervention with effective care and support are the key action points in these SCAR. However, knowledge and health-seeking behaviour for drug-induced allergies are multifactorial and are thought to differ between various communities. Continuous and repetitive community education may raise the public awareness of allergy and increase prompt health-seeking patterns in affected individuals.

\section{CONCLUSION}

Keeping in mind the significant morbidity and mortality associated with these SCAR, it would have been extremely beneficial if the culprit drug could be prevented. Proper elucidation of drug allergy history is imperative. If a patient is found to be allergic to a particular drug group, pharmacogenetic screening can be considered.

\section{Declaration of Patient Consent}

The authors certify that they have obtained all appropriate patient consent forms. The patient has given their consent for their images and other clinical information to be reported in the eJournal. The patient understands that their names and initials will not be published, and due efforts will be made to conceal their identity.

\section{References}

1. Harr T, French LE. Toxic epidermal necrolysis and Stevens-Johnson syndrome. Orphanet J Rare Dis. 2010;5:39.

2. Chan $\mathrm{HL}$ et al. The incidence of erythema multiforme, StevensJohnson syndrome, and toxic epidermal necrolysis. A populationbased study with particular reference to reactions caused by drugs among outpatients. Arch Dermatol. 1990;126(1):43-7.

3. Yang SC et al. The epidemiology of Stevens-Johnson syndrome and toxic epidermal necrolysis in China. J Immunol Res. 2018;2018:4320195.

4. Mockenhaupt $M$ et al. StevensJohnson syndrome and toxic epidermal necrolysis: assessment of medication risks with emphasis on recently marketed drugs. The EuroSCAR-study. J Invest Dermatol. 2008;128(1):35-44

5. Fakoya AOJ et al. Stevens - Johnson syndrome and toxic epidermal necrolysis; extensive review of reports of drug-induced etiologies, and possible therapeutic modalities. Open Access Maced J Med Sci. 2018;6(4):730-8.

6. Roujeau JC. The spectrum of Stevens-Johnson syndrome and toxic epidermal necrolysis: a clinical classification. J Invest Dermatol. 1994;102(6):28S-3OS.

7. Paumgartner $\mathrm{G}$, Beuers $U$. Ursodeoxycholic acid in cholestatic liver disease: mechanisms of action and therapeutic use revisited. Hepatology. 2002;36(3):525-31.

8. Ozkol HU et al. Ursodeoxycholic acid induced generalized fixed drug eruption. Cutan Ocul Toxicol. 2014;33(3):256-8.

9. Ellul JPM et al. Lichen planus associated with chenodeoxycholic acid and ursodeoxycholic acid for gallstone dissolution. Digest Dis Sci. 1992;37(4):628-30.

10. Su SC, Chung WH. Cytotoxic proteins and therapeutic targets in severe cutaneous adverse reactions. Toxins
(Basel). 2014;6(1):194-210.

11. Kumar R et al. Management of Stevens-Johnson syndrome-toxic epidermal necrolysis: looking beyond guidelines! Indian J Dermatol. 2018;63(2):117-24.

12. Horiuchi Y. Lichenoid eruptions due to ursodeoxycholic acid administration. Gastroenterology. 2001;121(2):P501-2

13. Tint GS et al. Ursodeoxycholic acid: a safe and effective agent for dissolving cholesterol gallstones. Ann Intern Med. 1982;97(3):351-6.

14. Ramam M et al. Oral drug provocation test to generate a list of safe drugs: experience with 100 patients. Indian J Dermatol Venereol Leprol. 2012;78(5):595-8.

15. Gupta LK et al. Guidelines for the management of StevensJohnson syndrome/toxic epidermal necrolysis: an Indian perspective. Indian J Dermatol Venereol Leprol. 2016;82(6):603-25. 